\title{
Individual Differences Reveal Correlates of Hidden Hearing Deficits
}

\author{
(D)Hari M. Bharadwaj, ${ }^{1,2}$ Salwa Masud, ${ }^{1,2}$ (E) Golbarg Mehraei, ${ }^{1,3}$ Sarah Verhulst, ${ }^{1,4}$ \\ and Barbara G. Shinn-Cunningham ${ }^{1,2}$ \\ ${ }^{1}$ Center for Computational Neuroscience and Neural Technology and 2Department of Biomedical Engineering, Boston University, Boston, Massachusetts \\ 02215, ${ }^{3}$ Program in Speech and Hearing Biosciences and Technology, Harvard University-Massachusetts Institute of Technology, Cambridge, \\ Massachusetts 02139, and ${ }^{4}$ Cluster of Excellence Hearing4all and Medizinische Physik, Department of Medical Physics and Acoustics, Oldenburg \\ University, D-26111 Oldenburg, Germany
}

Clinical audiometry has long focused on determining the detection thresholds for pure tones, which depend on intact cochlear mechanics and hair cell function. Yet many listeners with normal hearing thresholds complain of communication difficulties, and the causes for such problems are not well understood. Here, we explore whether normal-hearing listeners exhibit such suprathreshold deficits, affecting the fidelity with which subcortical areas encode the temporal structure of clearly audible sound. Using an array of measures, we evaluated a cohort of young adults with thresholds in the normal range to assess both cochlear mechanical function and temporal coding of suprathreshold sounds. Listeners differed widely in both electrophysiological and behavioral measures of temporal coding fidelity. These measures correlated significantly with each other. Conversely, these differences were unrelated to the modest variation in otoacoustic emissions, cochlear tuning, or the residual differences in hearing threshold present in our cohort. Electroencephalography revealed that listeners with poor subcortical encoding had poor cortical sensitivity to changes in interaural time differences, which are critical for localizing sound sources and analyzing complex scenes. These listeners also performed poorly when asked to direct selective attention to one of two competing speech streams, a task that mimics the challenges of many everyday listening environments. Together with previous animal and computational models, our results suggest that hidden hearing deficits, likely originating at the level of the cochlear nerve, are part of "normal hearing."

Key words: central auditory processing disorders; cochlear neuropathy; envelope-following response; frequency-following response; hidden hearing loss; temporal coding

\section{Introduction}

Clinical practice characterizes hearing loss through pure-tone threshold audiometry. However, a number of normal-hearing (NH) listeners complain of difficulty communicating, especially when there are competing sounds, noise, and room reverberation. When such listeners seek clinical treatment, they are labeled as suffering from obscure auditory dysfunction (Saunders and Haggard, 1992), King-Kopetzky syndrome (Zhao and Stephens, 1996), or, more generally, (central) auditory processing disorders (Chermak and Musiek, 1997). The nonspecific nature of these labels underscores how little we understand about the underlying mechanisms.

As many as $5-15 \%$ of adult patients seeking audiological help turn out to have normal thresholds (Cooper and Gates, 1991;

\footnotetext{
Received Sept. 21, 2014; revised Nov. 21, 2014; accepted Nov. 28, 2014

Author contributions: H.M.B., G.M., and B.G.S.-C. designed research; H.M.B., S.M., and S.V. performed research; H.M.B. and S.M. analyzed data; H.M.B., G.M., S.V., and B.G.S.-C. wrote the paper.

This work was supported by a fellowship from the Office of Assistant Secretary of Defense for Research and Engineering to B.G.S.-C.

Correspondence should be addressed to Hari M. Bharadwaj, Boston University Center for Computational Neuroscience and Neural Technology, 677 Beacon Street, Boston, Massachusetts 02215. E-mail: hari@nmr.mgh.harvard.edu.

DOI:10.1523/JNEUROSCI.3915-14.2015

Copyright $\odot 2015$ the authors $\quad 0270-6474 / 15 / 352161-12 \$ 15.00 / 0$
}

Kumar et al., 2007; Hind et al., 2011). Importantly, the problem may be even more common than such figures suggest; for instance, some NH listeners, particularly older participants, perform poorly on behavioral tasks that rely on precise temporal cues in suprathreshold sound (Strelcyk and Dau, 2009; Grose and Mamo, 2010; Ruggles et al., 2012). Auditory temporal perception degrades with age, independently of hearing loss and throughout adulthood (Snell and Frisina, 2000; Snell et al., 2002; Fitzgibbons and Gordon-Salant, 2010; Ruggles et al., 2012). Many listeners who can communicate effectively in quiet settings are nonetheless frustrated in complex environments such as cocktail parties, noisy restaurants, and busy streets (Dubno, 1984; Gatehouse and Noble, 2004). These findings suggest that a significant portion of listeners with normal or near-normal hearing thresholds exhibit temporal processing deficits.

Previous animal work revealed that noise exposure causing temporary threshold shifts, previously thought to be innocuous, can produce a loss of as many as $50 \%$ of the synapses and cochlear-nerve terminals innervating the inner hair cells, described herein as cochlear neuropathy (Kujawa and Liberman, 2009; Lin et al., 2011b). Aging also appears to reduce the effective auditory nerve fiber (ANF) population, initially through a loss of synapses with a subsequent degeneration of cell bodies (Makary 
et al., 2011; Sergeyenko et al., 2013). Furthermore, significant levels of deafferentation do not appear to affect detection thresholds (Schuknecht and Woellner, 1955; Lobarinas et al., 2013); however, in the healthy auditory system, convergence of nerve fibers is important for precise coding of both temporal finestructure and envelope cues in suprathreshold sounds (Joris et al., 1994; Lopez-Poveda and Barrios, 2013). Given the importance of temporal information for speech perception (Zeng et al., 2005), source localization (Blauert, 1997), grouping of acoustic elements into perceptual objects (Darwin, 1997; Shamma et al., 2011), and release from masking (Moore, 2008; Christiansen et al., 2013), degraded temporal coding is likely to interfere with suprathreshold perceptual ability — a form of "hidden hearing loss" (Bharadwaj et al., 2014; Plack et al., 2014).

Using a combination of electrophysiological, otoacoustic, and perceptual measures, the current study exploits individual differences in a cohort of $\mathrm{NH}$ listeners to characterize the relationships between peripheral function, neural temporal coding, and suprathreshold hearing performance with complex stimuli. We find that suprathreshold temporal coding fidelity varies in listeners with no known hearing complaints in a pattern consistent with cochlear neuropathy.

\section{Materials and Methods}

A primary goal of the study was to determine whether the pattern of individual differences in suprathreshold temporal coding in a cohort of young $\mathrm{NH}$ adult listeners is consistent with cochlear neuropathy. We explicitly evaluated peripheral cochlear mechanics and hair cell function to disambiguate where differences arise. Because noise-induced temporary and permanent threshold shifts often present as notches around 4 kHz (Pierson et al., 1994; Yost, 2007), our stimuli and testing focused on this frequency region.

Methods and measures were designed to imitate the challenges facing listeners in everyday, noisy environments in controlled laboratory settings. In contrast to listening to a single speech source in quiet (which is sparse in both time and frequency), acoustic scenes with multiple talkers, noise, and room reverberation typically have the following:

(1) High overall sound levels. A larger percentage of the overall population of ANFs will respond, including high-threshold, low-spontaneous rate (SR) fibers that would contribute less at low sound intensities.

(2) A relatively dense pattern of cochlear excitation along the tonotopic axis. "Off-frequency" ANF channels, remote from the dominant stimulus-evoked excitation locations, cannot encode target information. This is in contrast to listening in quiet where offfrequency high-SR fibers would respond to target modulations (Fig. 1).

(3) Relatively weak signal modulation. In addition to directly masking the target modulations, energy from competing sources will tend to fill in temporal dips in signal energy, requiring robust encoding of small modulations of high-intensity sounds for target information to be discernible (Fig. 1).

These observations suggest that at the level of the auditory nerve, degradations in temporal coding fidelity may be evident when listening in noisy real-world conditions even though they may not impair perception in conditions like those often tested in the laboratory. We therefore employed high sound levels, off-frequency maskers, and stimuli with shallow modulation depths in our battery of measures (Table 1). Given that neuropathy from both noise exposure and aging preferentially (though by no means exclusively) impacts low-SR fibers (Fig. 2), we hypothesized that such stimuli would increase the likelihood of exposing suprathreshold temporal coding deficits resulting from neuropathy (Bharadwaj et al., 2014). We measured peripheral/cochlear processing of sounds using distortion-product otoacoustic emissions (DPOAEs; Johnson et al., 2006), psychophysical tuning curves (Oxenham and Shera 2003), and audiometric detection thresholds. We measured early neural coding of temporal information using subcortical envelope-following responses (EFRs) (Bharadwaj and Shinn-Cunningham, 2014; Bharadwaj et al., 2014). We then compared these measures to perceptual estimates of temporal sensitivity, to electroencephalography (EEG)-based measures of cortical representation of acoustic spatial features (Salminen et al., 2010), and to selective attention performance in a complex speechon-speech masking task. Finally, we compared perceptual measures of temporal sensitivity to self-reported noise-exposure history.

All measures were obtained with the participants seated in an acoustically and electrically shielded booth (single-walled Eckel C-14 booth). For passive measures (DPOAEs and EFRs), participants watched a silent, captioned movie of their choice, ignoring the acoustic stimuli. A personal desktop computer controlled all aspects of the experiment, including triggering sound delivery and storing data. Special-purpose soundcontrol hardware (System 3 real-time signal processing systems, including analog-to-digital conversion and amplification; Tucker Davis Technologies) presented sound through insert phones (ER-1; Etymotic; for all measures except otoacoustic emissions, which used ER-10c) coupled to foam ear tips. All sounds were digitized at a sampling rate of $48,828 \mathrm{~Hz}$. For all active behavioral experiments, subjects responded by button presses. Feedback was presented after each trial.

\section{Participants}

Twenty six (13 female) subjects, aged 21-39 years, were recruited from the Boston University community. All subjects had pure-tone hearing thresholds better than $15 \mathrm{~dB}$ hearing level in both ears at octave frequencies between $250 \mathrm{~Hz}$ and $8 \mathrm{kHz}$ and rated their own hearing as normal and similar to others in their age group. In addition, two subjects (aged 27 and 29) with a "notch"-type hearing loss at $4 \mathrm{kHz}$ were recruited to evaluate the sensitivity of the measures used to characterize cochlear mechanical function. Subjects provided informed consent in accordance with protocols established at Boston University. Although the goal was to obtain each of the measures (Table 1) on every subject, subject availability precluded some subjects from completing some measures (see Fig. 4 , Fig. 6).

\section{Correlates of cochlear mechanical function}

Psychophysical tuning curves. An important aspect of healthy basilar membrane processing is good frequency selectivity. Psychophysical tuning curves were measured for a fixed, low-intensity probe tone (sensation level (SL) of $10 \mathrm{~dB}$ ) using the notched-noise method (Patterson, 1976) in a forward-masking paradigm (Oxenham and Shera, 2003). Stimulus parameters and testing procedures were identical to those used by Oxenham and Shera (2003). Filter equivalent rectangular bandwidths (ERBs) were estimated by fitting a $\operatorname{roex}(\mathrm{p}, \mathrm{w}, \mathrm{t})$ function (see Oxenham and Shera, 2003).

DPOAE input-output curves. To obtain an objective correlate of cochlear mechanical nonlinearity, DPOAE growth functions were measured as a function of level of the $\mathrm{f} 2$ primary tone ( $\mathrm{f} 2=4 \mathrm{kHz}$ ). The frequency and level of the $\mathrm{f} 1$ tone were varied according to the formula provided by Johnson et al. (2006) to maximize the level of the DPOAE for each level of the $\mathrm{f} 2$ tone. The DPOAE level was estimated at the distortion frequency of $2 \mathrm{f} 1-\mathrm{f} 2$.

\section{Behavioral measures of temporal coding}

Amplitude modulation (AM) detection thresholds were obtained using broadband noise stimuli (Viemeister, 1979). The target was a $500 \mathrm{~Hz}$ band of noise centered at $4 \mathrm{kHz}$ and modulated at $19 \mathrm{~Hz}$. Unmodulated bands of noise above (up to $10 \mathrm{kHz}$ ) and below (down to $20 \mathrm{~Hz}$ ) served as maskers to reduce off-frequency cues. The target to notched-noise ratio was $10 \mathrm{~dB}$, and the overall level was $80 \mathrm{~dB}$ SPL [broadband root mean square (RMS)]. Reference signals were statistically identical but without any amplitude modulation. The stimuli were presented diotically in a two-alternative, forced-choice paradigm (i.e., one interval with the reference signal and another with the target signal) for different modulation depths sampled randomly between 0 and $100 \%$. By using noise stimuli, spectral cues for AM detection were eliminated. Threshold depths for detection were determined using a Bayesian approach, fitting the parameters of a sigmoidal psychometric function to the responses from 1500 
A

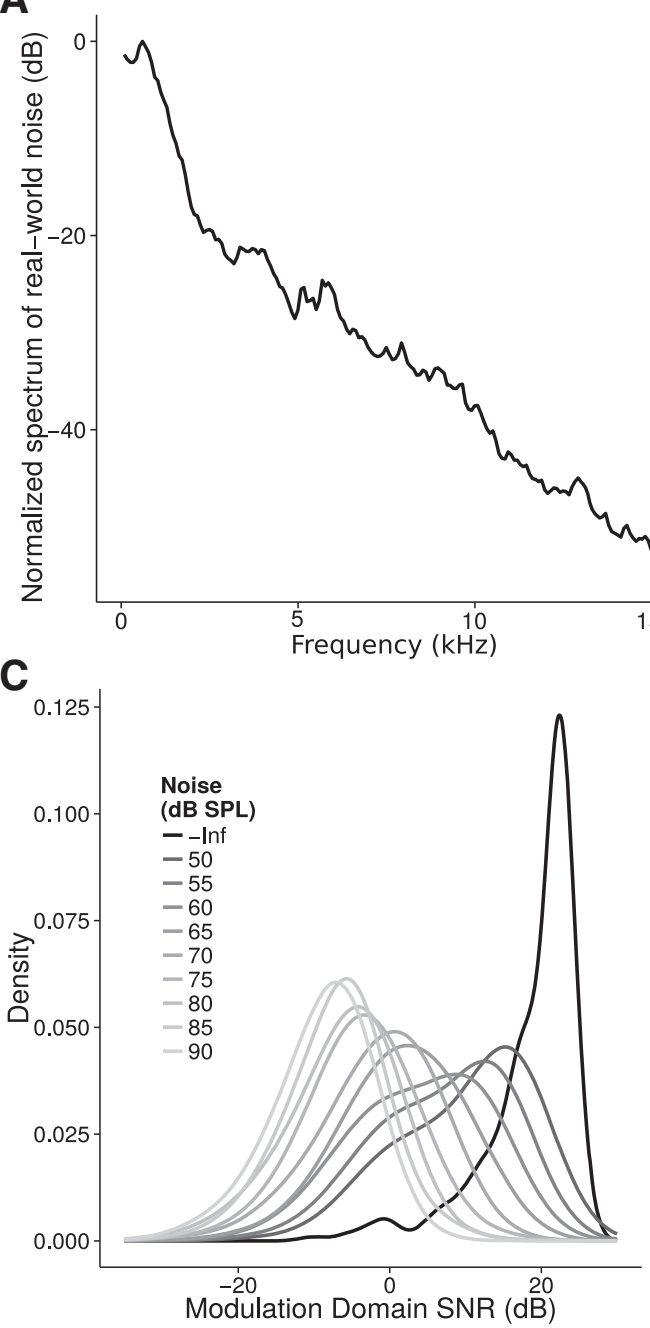

B

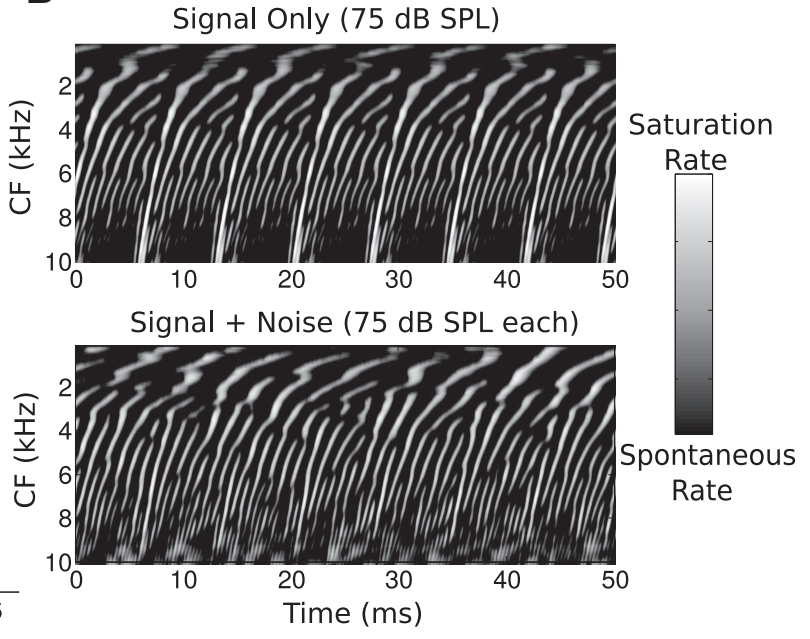

D

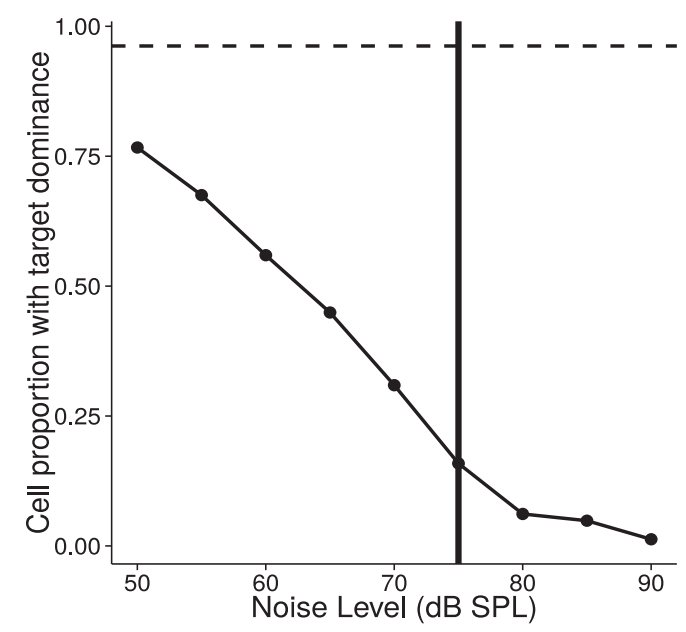

Figure 1. Illustration of the detrimental effects of real-world noise (restaurant chatter) on the representation of a target sound at the level of the auditory nerve (AN). $\boldsymbol{A}$, Long-term spectrum of noise recorded from a crowded restaurant showing the typical $1 / \mathrm{f}$ magnitude characteristic. $\boldsymbol{B}$, Representation of a target vowel (pitch, $140 \mathrm{~Hz}$ ) simulated using a phenomenological model of the AN (Zilany et al., 2014) in quiet (top) and with additive restaurant chatter (bottom) for a short analysis time window across fibers with different characteristic frequencies (CFs). Although strong modulations are apparent in both cases, the latter shows more irregularities. C, Distribution of the SNR across time-frequency "cells" (50 ms time windows for each cochlear channel). Within a given time-frequency cell, the SNR was defined as the ratio of the power in the modulation spectrum of the AN response at the target pitch $(140 \mathrm{~Hz})$ relative to the background. As more noise is added, the AN representation shifts from representing target modulations robustly ( $>20 \mathrm{~dB}$ SNR) to representing mostly noise (modulation SNR growing progressively negative). D, Proportion of timefrequency "cells" dominated by the target (i.e., having an SNR of $>3 \mathrm{~dB}$ ). Although $\sim 95 \%$ of the cells represent the target modulations in quiet (dotted line), when the noise level matches the signal level (solid vertical line), only $15 \%$ of the "cells" represent the target. Thus, although most time windows and most cochlear channels represent the target in quiet, maskers reduce both the temporal modulations and the availability of "off-frequency" channels.

Table 1. A summary of the list of measures used to characterize suprathreshold auditory perception and the corresponding stimulus used

\begin{tabular}{ll}
\hline Measure & Stimulus \\
\hline Audiograms & $250 \mathrm{~Hz}$ to $8 \mathrm{kHz}$ pure tones \\
Psychophysical tuning curves & $4 \mathrm{kHz}$ tones at $10 \mathrm{~dB} \mathrm{SL}$ in notched noise \\
DP0AE growth curves & $4 \mathrm{kHz}$ "f2" frequency at 16 levels \\
Modulation detection & Broadband noise with a $500 \mathrm{~Hz}$ band around $4 \mathrm{kHz}$ \\
& modulated at $19 \mathrm{~Hz}$ (80 dB SPL) \\
Envelope-ITD discrimination & $4 \mathrm{kHz}$ AM tones in notched noise modulated at $40 \mathrm{~Hz}$ \\
& (75 dB SPL) \\
Envelope-following & $4 \mathrm{kHz}$ AM tones in notched noise modulated at $100 \mathrm{~Hz}$ \\
responses & at a fixed level (75 dB SPL) and with varying modu- \\
lation depths (Fig. $5 \mathrm{~A})$
\end{tabular}

trials and calculating the posterior mean threshold using a Monte Carlo Markov chain (MCMC) sampling procedure from the posterior density (Kuss et al., 2005). As in Kuss et al. (2005), a normal and a log-normal prior were used for the location (threshold) and the slope parameters, respectively. The chance level was fixed at $50 \%$, and a $\beta$ prior was used for the lapse rate parameter.

To obtain a binaural measure of temporal coding, we used an envelope interaural time difference (ITD) discrimination task. A half-wave rectified sinusoid at $40 \mathrm{~Hz}$ was used to modulate a $4 \mathrm{kHz}$ carrier (called a "transposed" tone; Bernstein and Trahiotis, 2002). The envelope rate of $40 \mathrm{~Hz}$ was chosen because we acquired EEG data simultaneously with the task; this rate is known to elicit strong cortical source auditory steadystate responses (ASSRs) in EEG (Picton et al., 2003). The carrier phase was identical in the two ears; the ITD was applied only to the $40 \mathrm{~Hz}$ envelope. The envelope was ramped slowly (simultaneously in the two ears) over a $100 \mathrm{~ms}$ time window to minimize the use of onset cues. The stimulus level was set at $75 \mathrm{~dB}$ SPL. Off-frequency notched-noise (notch width of $800 \mathrm{~Hz}$ ) maskers, realized independently in each trial and uncorrelated across the two ears, were presented at a signal-to-noise ratio 
(SNR) of $10 \mathrm{~dB}$ (broadband RMS). The offfrequency masker extended to $20 \mathrm{~Hz}$ on the low-frequency side and $10 \mathrm{kHz}$ on the highfrequency side and served to attenuate offfrequency listening cues, including cues from distortion products. Each trial consisted of a sequence of two intervals, with the second interval having an envelope ITD of zero. The stimulus in the first interval had an ITD sampled uniformly from the set $\{50,100,200,400$, $800\} \mu \mathrm{s}$, with the leading ear randomized across trials. In each trial, the subjects were asked to indicate whether the sound moved from "left to center" or "right to center." As with the AM threshold measurement, the threshold was determined by fitting a sigmoidal psychometric function to responses from 1200 trials and estimating the posterior mean using MCMC sampling. For the small number of subjects whose thresholds appeared to exceed $800 \mu$ s, additional trials were included with ITDs up to $1200 \mu \mathrm{s}$.

\section{Electrophysiological measures of temporal coding}

EFRs were measured in response to 400 -ms-long bursts of $100 \mathrm{~Hz}$ transposed tones with a carrier frequency of $4 \mathrm{kHz}$ at a level of $75 \mathrm{~dB}$ SPL and for varying modulation depths $(m)$. EFRs were obtained using a 32 channel EEG system (Biosemi Active II system) using 1000 presentations of each stimulus with half in each polarity. The interstimulus interval varied uniformly between 550 and $650 \mathrm{~ms}$. The EFR power was estimated in the frequency domain using a complex principal component analysis approach to combine across channels. This approach combines measurements across multiple channels, optimally adjusting for phase disparities in the signal across channels; the approach has been shown to improve the SNR of the extracted EFR significantly (Bharadwaj and ShinnCunningham, 2014). Off-frequency notched-noise maskers were applied at an SNR of $20 \mathrm{~dB}$ (broadband RMS) with a notch width of $800 \mathrm{~Hz}$ to attenuate off-frequency contributions. The envelope rate and noise level were fixed based on pilot experiments requiring a large number of trials. These pilot experiments showed that at low noise intensities, small changes in noise level had a stronger effect, whereas at moderate noise levels, decreases in SNR had more modest effects; there was a knee point at an SNR of $\sim 20 \mathrm{~dB}$. The pilot experiments also showed that at an envelope rate of $100 \mathrm{~Hz}$, robust responses could be acquired and exhibited a group delay of $\sim 8 \mathrm{~ms}$, consistent with purely subcortical generators (Kiren et al., 1994; Herdman et al., 2002; Kuwada et al., 2002). The off-frequency noise was realized independently in each trial and was interaurally uncorrelated.

We hypothesize that cochlear neuropathy underlies a portion of individual differences in temporal coding fidelity. Modeling the EFR as a summed response over a population of (model) inferior colliculus cells, Bharadwaj et al. (2014) suggested that (1) stimuli at high levels and shallow modulation depths should accentuate the contributions of low-SR fibers, and that (2) changes in EFR amplitude with modulation depth would provide an easy-to-interpret, self-normalized measure of suprathreshold coding fidelity.

EFRs were recorded for six different modulations [ $m$ values with 20 $\log (m)$ varying from $100 \%$ modulation or $0 \mathrm{~dB}$ up to $-20 \mathrm{~dB}$ in steps of $-4 \mathrm{~dB}$. A multichannel estimate of the phase-locking value (PLV) was used to infer whether an EFR peak exceeded the noise floor. The PLV is convenient for this purpose because the noise floor distribution is independent of background noise levels (Zhu et al., 2013; Bharadwaj and Shinn-Cunningham, 2014). Because half of the subjects did not show a significant EFR peak at the modulation frequency of $100 \mathrm{~Hz}$ for modulation depths of $-12 \mathrm{~dB}$ and lower, only the EFRs in response to stimuli with the largest modulations were analyzed. As suggested by (Bharadwaj et al., 2014), the slope of the EFR amplitude with modulation depth was used to quantify suprathreshold coding fidelity, computed by fitting a straight line over the three data points corresponding to $20 \log (m)=0$, -4 , and $-8 \mathrm{~dB}$, respectively.

Cortical EEG was recorded simultaneously with the envelope-ITD detection task to obtain an electrophysiological correlate of binaural temporal sensitivity and to assess if early neural coding affects the cortical representation of acoustic features. Adaptation of the cortical onset response ("N100 response") to the second of a pair of sound bursts is ITD specific; the response to the second sound is larger when it differs in ITD from the first (Salminen et al., 2010). We wondered whether such ITD-specific adaptation can be found when the ITD is purely in the envelope, as in our behavioral task (see Fig. 7A). The $40 \mathrm{~Hz}$ cortical ASSR was also quantified.

\section{ITD-based selective attention task}

To evaluate the contributions of individual differences in cochlear mechanical function and suprathreshold temporal coding to differences in listening performance in a complex task, a "spatial" attention task was used. Spoken digits recorded in-house in the voice of a female speaker were monotonized to $184 \mathrm{~Hz}$ (close to the natural pitch of the voice). To simulate some of the constraints imposed by realistic maskers, uncorrelated pink noise (1/f spectrum; Fig. $1 A$ ) was added to each ear. The signals were spatialized by imposing simple ITDs. Each trial consisted of two simultaneous sequences of three spoken digits each, with the streams differentiated only by their ITDs, which were symmetrical around the midline (see Fig. 8A). A visual cue presented $2 \mathrm{~s}$ before the onset of the sound streams identified the direction of the target stream (left or right). The ITD magnitudes in each trial were drawn uniformly from the set 50 , $100,200,300,400 \mu \mathrm{s})$. The target direction was randomized on each trial. At the end of each trial, a visual response circle cued the subjects to indicate the target using a sequence of button presses. The effects of cochlear mechanical function and suprathreshold temporal coding fidelity on performance in the attention task were determined using a generalized linear regression analysis (Pinheiro and Bates, 2000). Subject-specific residuals were modeled as random effects coming from a normal distribution. Normal priors were used for each location parameter (model coefficients), and inverse-Wishart priors were used for variance components (Hadfield, 2010).

\section{Results}

\section{Correlates of peripheral processing}

Figure $3 A$ shows the DPOAE growth function for subjects in our $\mathrm{NH}$ cohort (black), as well as for two subjects with hearing loss (red lines). The standard error of the mean over the NH cohort is also shown for reference. The results are comparable to published DPOAE input-output curves for $\mathrm{NH}$ listeners (Johnson et al., 

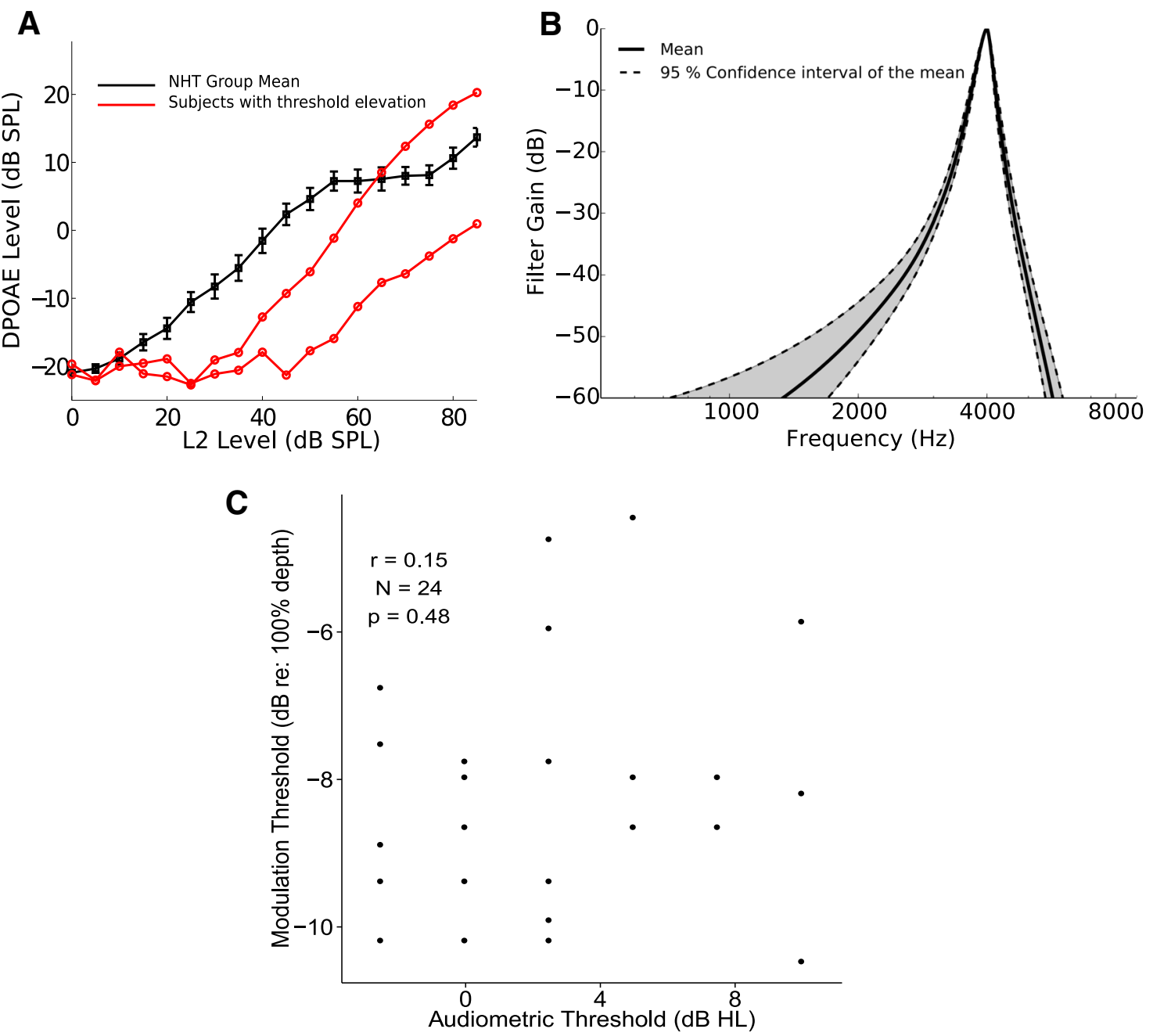

Figure 3. Objective and behavioral measures of cochlear hair cell function. $A$, DPOAE input- output curves ( $f 2=4 \mathrm{kHz}$ ) for the NH cohort and two subjects with elevated thresholds. DPOAE thresholds are elevated for subjects with hearing loss, affirming that the measure is sensitive to cochlear mechanical deficits. $\boldsymbol{B}$, Forward-masking tuning curves at $10 \mathrm{~dB}$ SL for the NH cohort. C, Comparison of audiometric thresholds and modulation thresholds across individuals. There is no significant correlation.

2006). The relatively large attenuation in the DPOAE for the two subjects with hearing loss testifies to the sensitivity of the measure to dysfunction in cochlear mechanics. We defined the DPOAE threshold for each subject as the lowest presented level of the $\mathrm{f} 2$ primary tone at which the DPOAE level exceeded - $10 \mathrm{~dB}$ SPL. These DPOAE thresholds were then compared with behavioral audiometric thresholds and with the behavioral measures of suprathreshold temporal perception. Although all of the listeners in our NH cohort have thresholds within the normal range (by design), there are modest residual differences in the thresholds across listeners (see horizontal distribution of data in Fig. $3 C$ ). Psychophysical filter widths also were comparable to those of $\mathrm{NH}$ listeners in the published literature (Oxenham and Shera, 2003), with a mean ERB of $249 \mathrm{~Hz}$ and a standard error of $24 \mathrm{~Hz}$ (Fig. $3 B)$. Importantly, the three measures of cochlear amplifier function correlated with each other (ERBs vs pure-tone thresholds, $r=0.51, p=0.011$; DPOAE vs pure-tone thresholds, $r=0.42$, $p=0.04$ ), suggesting that the variance in these measures across subjects is not dominated by measurement noise, but rather reflects individual differences in cochlear function among the listeners in the $\mathrm{NH}$ cohort.

\section{Behavioral correlates of temporal sensitivity}

Similar to previous reports of large individual differences in temporal coding in NH listeners (Ruggles et al., 2011), we found large individual differences in AM thresholds across our $\mathrm{NH}$ cohort (see vertical distribution of data in Fig. $3 C$ ). The measured thresholds are higher than AM thresholds for noise stimuli reported in the literature (Viemeister, 1979); this discrepancy is likely due to the fact that our narrowband modulation target signal was presented with unmodulated off-frequency bands of masking noise at a high spectral level. Crucially, these differences in AM thresholds appear to be unrelated to the small individual differences in pure-tone thresholds at $4 \mathrm{kHz}$ (Fig. 3C).

Concomitantly, we also found large individual differences in binaural measures of temporal coding. As shown in Figure 4, envelope-ITD sensitivity for a carrier frequency of $4 \mathrm{kHz}$ varies over a wide range across listeners, from 6 to $19 \mathrm{~dB}$ relative to 100 $\mu$ s (i.e., from $\sim 200$ to $900 \mu \mathrm{s}$ ). This range is greater and the absolute threshold values higher than typical past reports (Bernstein and Trahiotis, 2002); however, in contrast to most past studies, we presented our stimuli along with relatively high intensity, interaurally uncorrelated off-frequency noise maskers (Bernstein and Trahiotis, 2008). Across listeners, neither mea- 


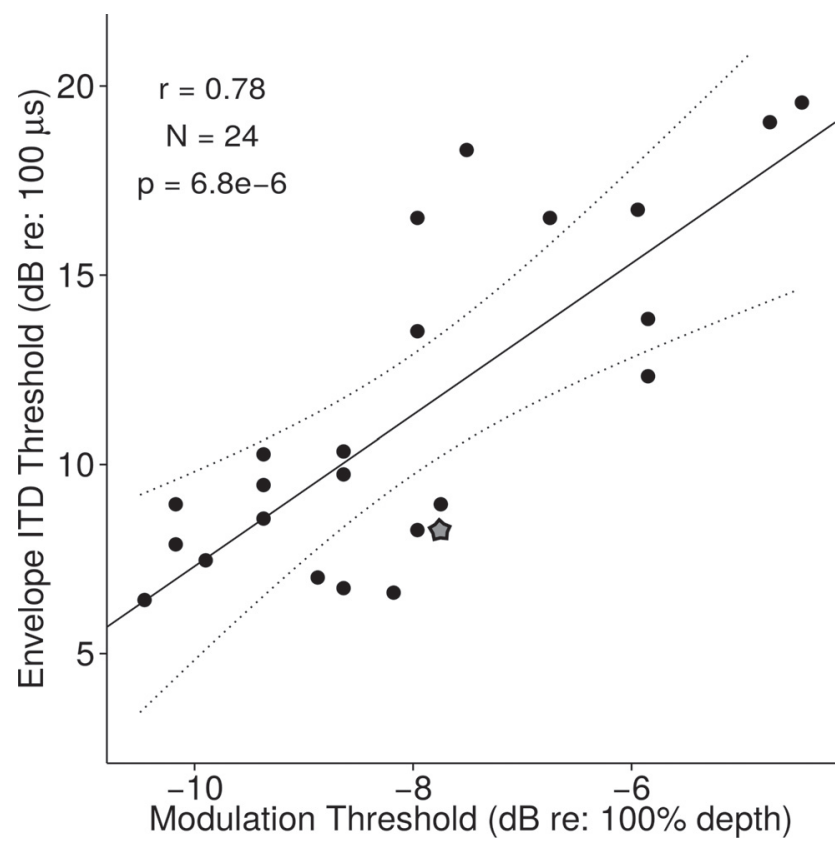

Figure 4. Relationship between monaural (modulation thresholds) and binaural (envelope ITD thresholds) measures of temporal sensitivity. The subject denoted with the star symbol was unavailable for the EFR measures. A considerable portion of the variance in the binaural measure can be accounted for by differences in the "monaural" measure.

sure of temporal coding fidelity correlated with audiometric threshold measurements; however, the two measures were significantly correlated with each other $(r=0.78, p=6.8 \mathrm{e}-6$; Fig. 4). Similarly, psychophysical tuning curve bandwidths (ERBs) did not correlate with either AM thresholds $(r=0.11, p>0.05)$ or envelope-ITD thresholds $(r=0.21, p>0.05)$ at the $5 \%$ falsealarm-rate level.

\section{Subcortical neural correlates of temporal coding}

To assess temporal coding precision with a physiological measure, EFR magnitudes were extracted using frequency-domain principal component analysis (Bharadwaj and Shinn-Cunningham, 2014). Figure $5 B$ shows the EFRs obtained for the four deepest modulation depths tested. On average, as the modulation depth decreases, the EFR magnitude decreases. Figure $5 B$ also shows traces for a representative sampling of the individual $\mathrm{NH}$ subjects (the first 10 enrolled in the study, in chronological order). Whereas some individuals showed a gradual decrease in the EFR magnitude as modulation depth decreases, others showed a more precipitous decrease leading to weak EFR responses at shallow modulation depths. For each subject, we fit a straight line to the EFR magnitudes obtained with the three largest modulation depths $(0,-4$, and $-8 \mathrm{~dB})$ and considered the slope relating EFR magnitude to modulation depth. As shown in Figure $5 B$, slopes varied from close to $0 \mathrm{~dB}$ EFR change per $4 \mathrm{~dB}$ of modulation to $12 \mathrm{~dB}$ EFR change per $4 \mathrm{~dB}$ of modulation. These slopes correlate significantly with behavioral AM thresholds (Fig. 6A). Subjects whose EFR magnitude dropped precipitously with a decrease in input modulation had poorer AM sensitivity (high AM thresholds). For comparison, we also considered the absolute EFR magnitude at a shallow modulation depth (Fig. 6B). These absolute magnitudes also correlated significantly with behavioral AM thresholds (e.g., EFR at $-4 \mathrm{~dB}$ vs AM thresholds, $r=0.53, p=$ 0.008); however, the slope of EFR strength versus stimulus modulation depth exhibited a stronger correlation with the perceptual thresholds $(r=0.68, p=2.5 \mathrm{e}-4)$. To compare the correlations, we used the T2 test, which has been shown to perform well when comparing dependent correlations (Steiger, 1980). Based on this test, the slope metric was a significantly stronger correlate of AM thresholds than the EFR at any single modulation depth ( $p=$ 0.03). The EFR slope metric also correlated strongly with the binaural measure of temporal coding (envelope-ITD thresholds, $r=0.54, p=0.006$; Fig. $6 C$ ). That the EFR slope metric is a strong correlate of suprathreshold temporal sensitivity conforms to the model predictions of simulated low-SR ANF neuropathy (Bharadwaj et al., 2014). These correlations remained strong when other potential covariates were also included as predictors; specifically, a multiple regression model for the AM thresholds revealed that only the EFR slope was a significant predictor $\left(F_{(1,18)}=14.3, p=\right.$ $0.0013)$, whereas age $\left(F_{(1,18)}=0.007, p=0.95\right)$, $\operatorname{sex}\left(F_{(1,18)}=0.08\right.$, $p=0.77)$, and audiometric thresholds $\left(F_{(1,18)}=0.5, p=0.49\right)$ were not. Note, however, that our age range was narrow, with the distribution skewed toward the younger side (only three subjects were older than 30).

\section{Cortical EEG correlates of binaural temporal processing}

To explore whether the cortical representation of sound features is affected by the differences in subcortical coding fidelity, we acquired EEG data as listeners performed the envelope-ITD detection task (for the timing of the sequence of events constituting a trial, see Fig. $7 A$ ). Both the induced power (Fig. $7 B$ ) and the phase-locking (Fig. 7C) spectra show responses locked to the onset of the notched noise, which starts $400 \mathrm{~ms}$ before the target modulated tones. The late sustained responses in the induced power (after $1500 \mathrm{~ms}$ ) are likely related to the motor preparation for the button press response. Although the transposed tones do not induce a change in power compared to baseline, there is a clear phase-locked onset response in the low frequencies $(<20$ $\mathrm{Hz}$ ). In addition, both the variable and standard tones elicit ASSRs phase locked to the $40 \mathrm{~Hz}$ stimulus modulation frequency (Fig. 7C; onsets at 0 and $0.8 \mathrm{~s}$, respectively).

In general, the MEG/EEG onset response to the second ("probe") of a pair of identical stimuli is smaller than to the first (the "adaptor"), a result typically attributed to adaptation (Jääskeläinen et al., 2004). When the adaptor differs from the probe in one or more attributes, the response to the probe is less adapted/suppressed (Jääskeläinen et al., 2004). We used this fact to test cortical sensitivity to changes in ITDs. We explored how the response to a standard probe tone with 0 ITD depended on the ITD of a preceding variable-ITD adaptor. To quantify the adaptation of cortical onset responses, we computed the aggregate of the phase-locked EEG response from 0 to $20 \mathrm{~Hz}$. We did this separately for trials where the envelope ITD in the adaptor was large (400 and $800 \mu \mathrm{s}$; greater than the median threshold) versus when it was small $(50,100$, and $200 \mu \mathrm{s}$; smaller than the median threshold; see Fig. 7D). We found that when the ITD of the adaptor was small (i.e., more similar to the probe), the response to the subsequent 0 -ITD probe was smaller than when the ITD of the adaptor was large (i.e., more dissimilar to the probe; paired $t$ test, $t=2.8, p=0.01)$. This difference is a form of envelope-ITD-specific adaptation similar to what has been reported previously for stimuli with broadband ITDs (Salminen et al., 2010). For each individual, we computed the "adaptation ratio" by computing the ratio of the probe onset response when preceded by a large-ITD adaptor compared to a small-ITD adaptor. A weak but statistically significant negative correlation was found between the envelope-ITD threshold and the adaptation ratio across individuals $(r=-0.34, p=0.04)$. In other words, 
A

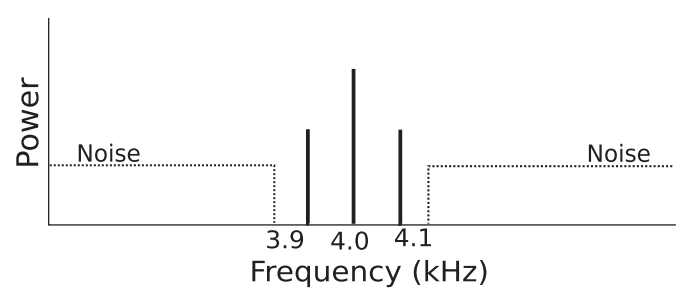

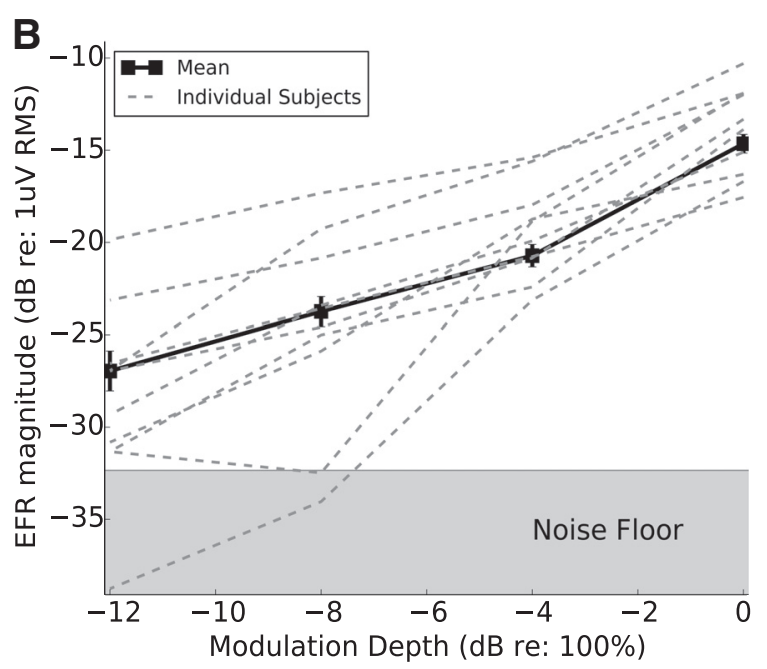

Figure 5. Results of subcortical steady-state response measures. $A$, lllustration of the stimulus used for EFR measures. The use of off-frequency noise attenuates contributions of tonotopic regions away from $4 \mathrm{kHz}$. $\boldsymbol{B}$, Large individual differences are observed in the EFR, particularly at lower modulation depths. Whereas some individuals show strong responses even for shallow modulations, others show a rapid decline in the early neural representation of envelopes as the modulation depth decreases.

listeners who have relatively poor sensitivity and large behavioral thresholds tend to have small onset responses to the standard tone preceded by a large-ITD tone, as if for them the large-ITD variable tones are perceptually similar to the 0 -ITD standard tone. These results suggest that subcortical temporal coding fidelity varies across NH listeners, affecting both behavior and cortical physiology.

In contrast with the $100 \mathrm{~Hz}$ EFR, analysis of the $40 \mathrm{~Hz}$ cortical ASSR revealed that there were considerable across-subject differences in the background cortical activity around the $40 \mathrm{~Hz}$ region. This may be due to anatomical factors, task-related background cortical activity, as well as other factors. Thus, we did not pursue any further analysis of this response.

\section{Relationship between attention task performance and correlates of cochlear mechanics and suprathreshold temporal coding}

Listeners reported the content of one of two simultaneous streams of spoken, monotonized digit sequences based on direction, which was controlled by imposing different ITDs on the streams (Fig. 8A). Results revealed significant individual differences, with performance ranging from chance level to ceiling (Fig. 8B). Errors generally arose because listeners reported the masker instead of the target, not from lapses in intelligibility or memory; specifically, $91 \%$ of the incorrectly reported digits appeared in the masking stream at the same position in the sequence. This suggests that sensory encoding limitations made it difficult to spatially resolve the target and masker, leading to attentional selection errors. To evaluate the relative contributions of peripheral processing and neural temporal coding to individual differences in the spatial attention task, we entered the data into a generalized linear regression analysis. Performance (number of correct responses) was modeled as coming from a binomial distribution. The latent binomial parameter was modeled through a logit link function using a linear mixed model (Hadfield, 2010) with ITD, individual EFR slopes, and DPOAE thresholds as explanatory variables. Inference was performed using MCMC sampling from the posterior. The coefficient of the DPOAE term was not distinguishable from zero, whereas the EFR term was significant $(p=0.0024)$. The marginal posterior of the coefficient of the EFR term was unimodal, with a 95\% highestposterior-density interval (Box and Tiao, 2011) spanning -5.9 to $0.95 \%$ with a mean of $-3.5 \%$; that is, for every $1 \mathrm{~dB} / 4 \mathrm{~dB}$ steepening of the EFR slope, the probability of a correct response dropped by an average of 3.5\%. Not surprisingly, the effect of ITD was also significant $(p=0.004)$, suggesting, along with the error patterns, that sensory limitations were a key determinant of performance. Thus, for this particular spatial attention task, individual differences in temporal coding accounted for much of the variance in performance.

\section{Noise-exposure history}

To get a sense of the relationship between noise exposure and subcortical temporal coding, we asked our subjects to rate their own exposure on a closed scale. Exposure history was assessed by considering the frequency that listeners reported attending concerts, loud parties, and sports venues and using earphones. In addition, subjects indicated whether they recalled ever having temporary threshold shifts ("losing sensitivity to faint sounds for a while") following noise exposure. Subjects were divided into two groups ("more exposed" and "less exposed") based on their responses, with equal weight given to all categories of exposure. Compared to the less exposed group, the more exposed group had a significantly steeper EFR slope $\left(t_{(23)}=1.92, p=0.034\right)$ along with higher AM $\left(t_{(23)}=1.97, p=0.03\right)$ and envelope-ITD $\left(t_{(23)}=2.35, p=0.014\right)$ thresholds compared to the less exposed group. These effects remain marginally significant when the false discovery rate for multiple comparisons is controlled at 5\% (Benjamini and Hochberg, 1995). Given the relatively crude characterization of noise-exposure history, correlations between all the temporal coding measures, and the size of our cohort, this result should to be interpreted with caution.

\section{Discussion}

In the conventional view, hearing loss is associated with cochlear mechanical anomalies, typically caused by hair cell loss or damage to mechanosensory hair bundles (for a detailed treatment, see Moore, 2007). In contrast, previous animal studies showed that moderate noise exposure and early aging can produce synapse/ neuronal loss without changes to cochlear mechanics and with no 
A

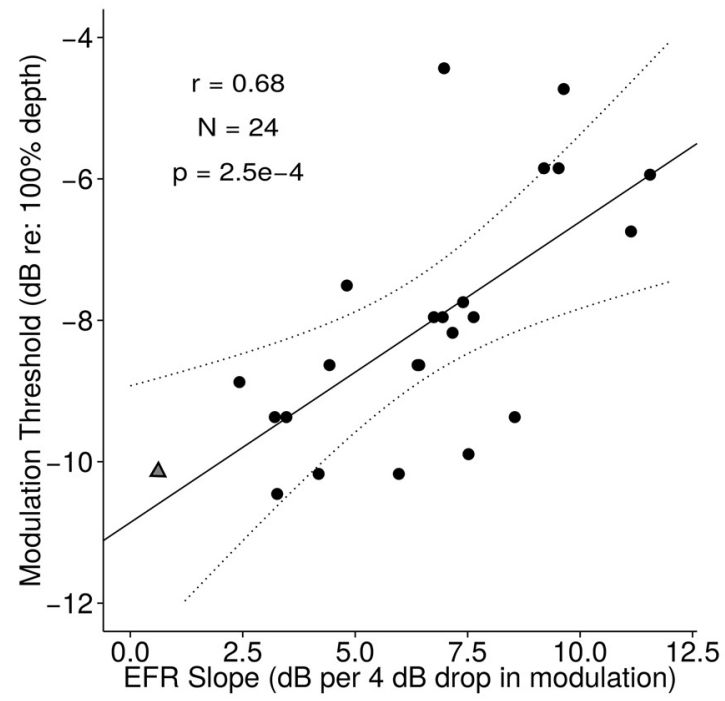

B

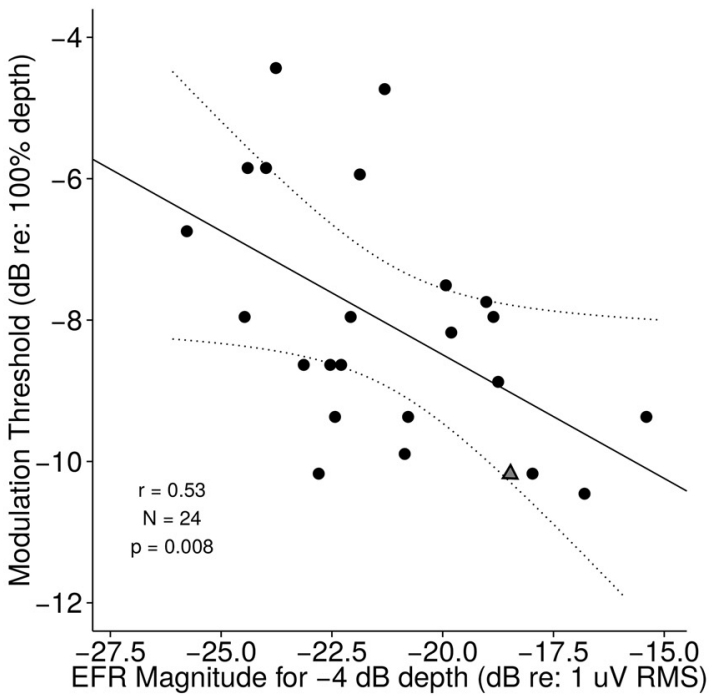

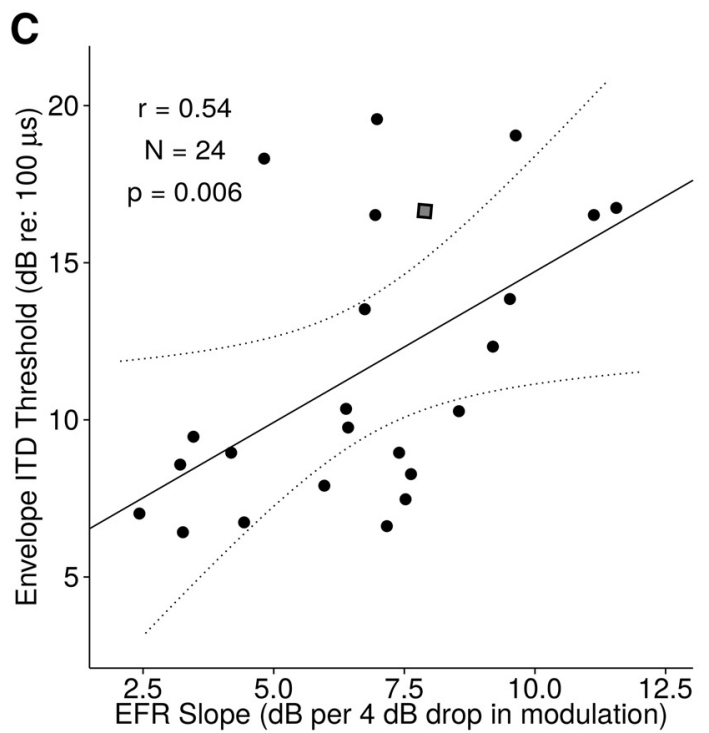

Figure 6. Relationship between EFR and behavioral measures of temporal sensitivity. A, AM thresholds versus EFR slope. The rate at which the EFR drops with decreasing modulation correlates strongly with perceptual modulation sensitivity. B, AM thresholds versus absolute EFR magnitude at $-4 \mathrm{~dB}$ modulation depth (the shallowest depth at which $90 \%$ of the subjects showed a clear EFR response distinguishable from the noise floor). Although the absolute EFR magnitude at a given modulation depth correlates with perceptual ability, the correlation is not as strong as the correlation between EFR slope and perception. C, Envelope ITD threshold versus EFR slope. Subcortical temporal coding fidelity as measured by EFR slope also can explain a considerable amount of the variance in envelope ITD thresholds. The subject denoted by the triangle symbol in $\boldsymbol{A}$ and $\boldsymbol{B}$ was unavailable for envelope ITD threshold measurement, and the subject denoted by the square symbol in $\boldsymbol{C}$ was unavailable for modulation threshold measurement.

hair cell loss - a completely different form of sensorineural hearing loss (Kujawa and Liberman, 2009; Makary et al., 2011; Sergeyenko et al., 2013).

Here, we investigated whether perceptual differences in the general population of $\mathrm{NH}$ listeners who have no known hearing deficits are consistent with this mechanism of synaptopathy/neuropathy (Bharadwaj et al., 2014). Our measures were designed to reveal differences in suprathreshold coding fidelity, using stimuli with relatively high sound levels, competing maskers, and subtle temporal features. These choices were important for two reasons: (1) temporal coding deficits that are not apparent in ordinary laboratory conditions may be exposed under such more challenging regimes (Fig. 1), and (2) low-SR fibers, which are especially vulnerable to neuropathy (Fig. 2) are likely to contribute more under such conditions. Specifically, compared to high-SR fibers, the low-SR ANFs are more resistant to masking (Costalupes,
1985), and exhibit more robust synchrony to both envelope and fine structure in high-intensity sounds (Johnson, 1980; Joris and Yin, 1992). To help isolate where differences arise, we evaluated peripheral transduction, early neural coding, cortical sensitivity to ITDs, and suprathreshold temporal perception. We found large variations in perceptual ability on both low-level psychophysical tasks and a high-level selective attention task; across subjects, these behavioral measures strongly correlated with physiological measures of suprathreshold temporal coding. Furthermore, we established that differences in cortical sensitivity of binaural information inherit some of the differences in the fidelity of early neural temporal coding. Crucially, independent measures of cochlear amplifier function, though correlated with each other, accounted for very little of the variability in attention task performance and were uncorrelated with psychophysical and physiological measures of suprathreshold temporal coding. Together, 
A

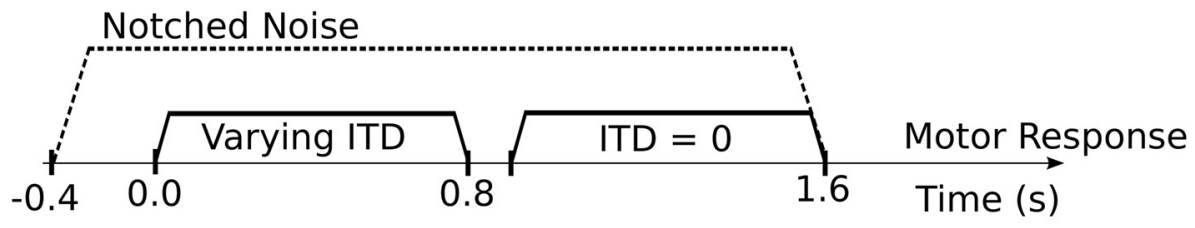

B

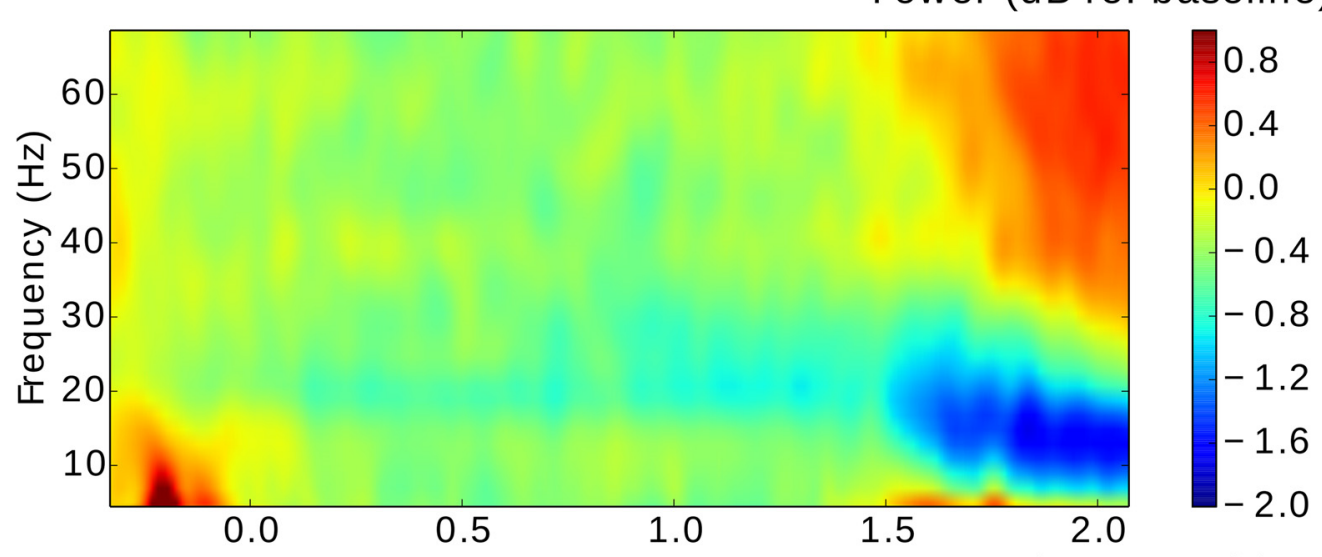

C
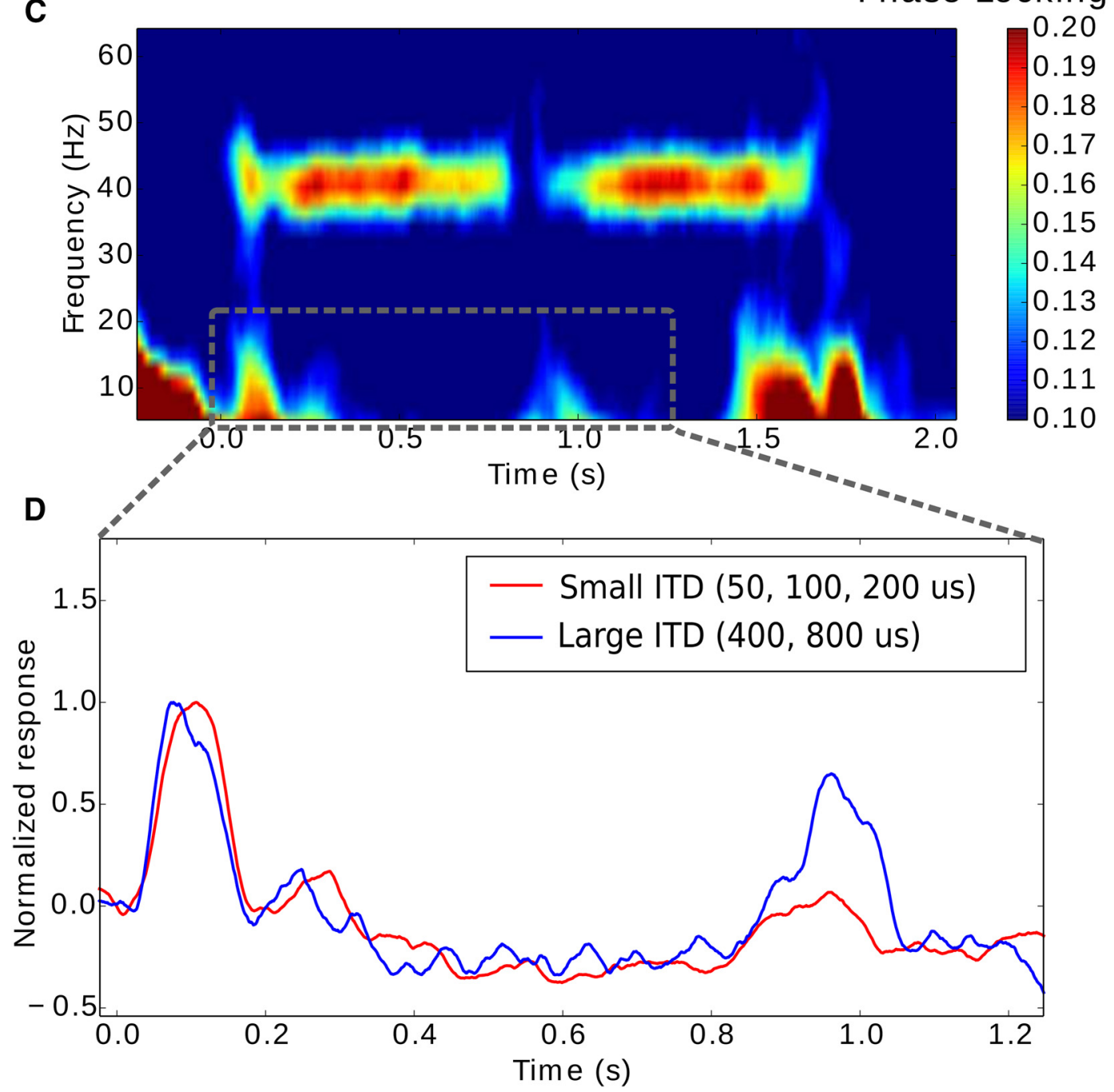

Figure 7. Results from cortical EEG measures of envelope ITD sensitivity. $\boldsymbol{A}$, Stimulus sequence. Subjects were asked to indicate if sounds jumped from left to center or right to center. $\boldsymbol{B}$, Induced power relative to baseline. $\boldsymbol{C}$, Intertrial phase locking reveals low-frequency responses to the onset of AM tones in addition to showing a sustained response at the modulation frequency ( $40 \mathrm{~Hz}$ ). $\boldsymbol{D}$, A closer look at the low-frequency power in onset responses to the two tones (averaged over frequencies below $20 \mathrm{~Hz}$ ) showing envelope ITD-specific adaptation. 

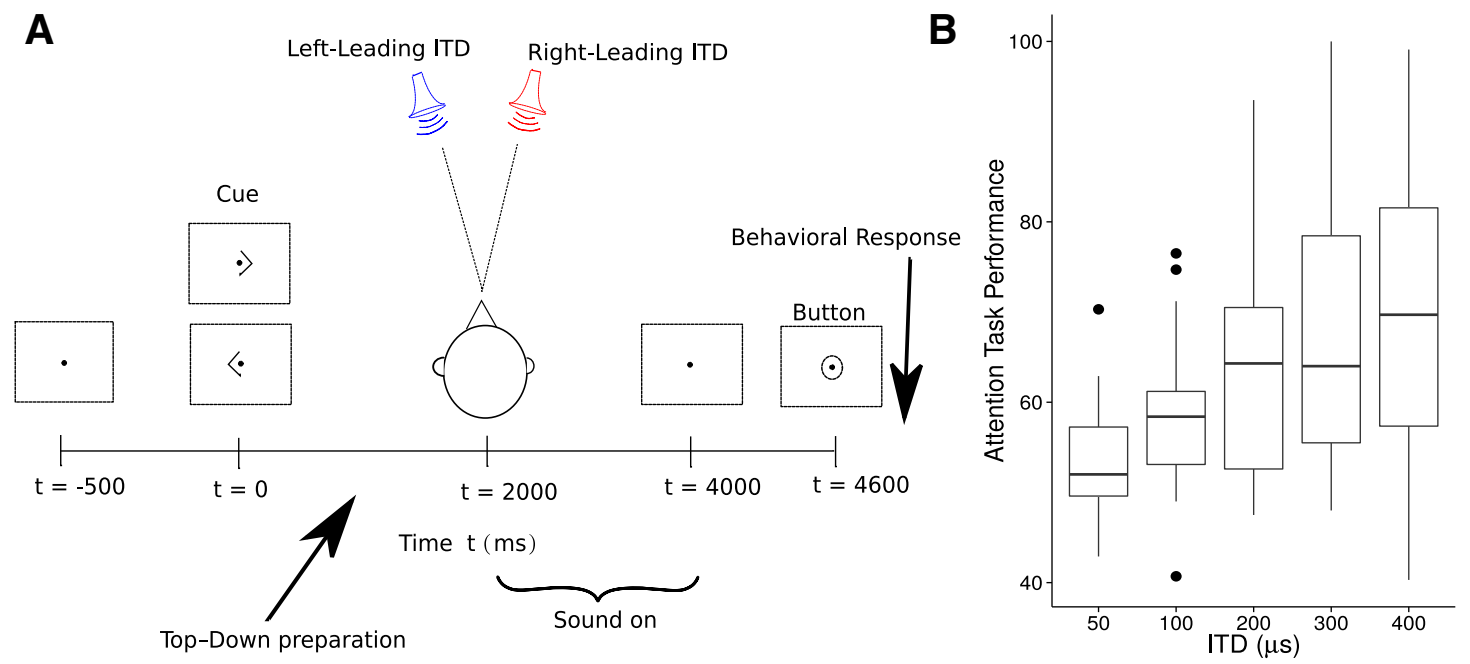

Figure 8. ITD-based attention. A, A schematic illustration of the sequence of events constituting the task. Each trial began with the subject visually fixated on the center of the screen. A visual cue (left or right arrow) appeared 2 s before the onset of the sounds, identifying the direction of the target stream (left or right, based on ITDs). Two simultaneous sequences of digits spoken by the same speaker and monotonized to the same pitch were then presented. Following the digit sequences, a visual response circle cued the subject to respond and indicate the three digits in the target sequence using button presses. Finally, feedback was given to the subject as follows: a green circle, indicating that all three digits were identified correctly; a blue circle, indicating that two of the three digits were identified correctly; or a red cross, indicating that fewer than two response digits matched the correct target sequence. $\boldsymbol{B}$, Performance as a function of ITD. Large individual differences are evident. The upper and lower hinges correspond to the first and third quartiles (the 25th and 75th percentiles). The upper whisker extends from the hinge to the highest value that is within $1.5 *$ IQR of the hinge, where IQR is the inter-quartile range, or distance between the first and third quartiles. The lower whisker extends from the hinge to the lowest value within $1.5 *$ IQR of the hinge. Data beyond the end of the whiskers are outliers and plotted as individual points. The drop in performance with ITD indicates that sensory coding limitations, perhaps related to source separability, dominate performance.

these results demonstrate that individual differences in NH listeners are in part driven by differences originating very early in the neural (and not mechanotransduction-related) portions of the auditory pathway.

To quantify physiological coding strength, we settled on the slope of the EFR as a function of modulation depth. Whereas absolute EFR strength (Fig. 6B) also correlated with behavioral performance, the EFR slope proved to be more a sensitive correlate of perceptual ability. This is likely both because the EFR slope (1) helps normalize differences in EFR magnitude due to individual differences in measurement noise, brain tissue geometry, and other phenomena leading to purely linear transformations of the responses, and (2) emphasizes coding fidelity of shallow stimulus modulations at high sound levels, where low-SR ANFs likely contribute significantly to coding (Bharadwaj et al., 2014). Consistent with the interpretation that differences are present as early as at the level of the auditory nerve, the EFR slope correlated with sensitivity to both monaural and interaural temporal information. Furthermore, the efficacy of the EFR slope in exposing individual differences is in accordance with a previous computational model of low-SR neuropathy (Bharadwaj et al., 2014). Of course, unlike in animal models, we cannot manipulate or measure cochlear neuropathy in humans directly; our interpretation is based on indirect evidence. Still, the observed pattern of results is consistent with that mechanism.

We found robust correlations across various measures of temporal coding, but no correlation between measures of cochlear mechanical function and perceptual abilities. Of course, if a larger cohort of $\mathrm{NH}$ participants were tested, residual differences in cochlear mechanics could well explain a significant portion of the individual variability. Regardless, our results raise the possibility that noise-induced neuropathy is a significant contributor to what has until now been viewed as "normal" variability in perceptual hearing ability. Indeed, an estimated $17 \%$ of the general population experience occupational noise at levels sufficient to produce temporary threshold shifts and have subsequent hearing difficulties that are attributable to this exposure (Tak and Calvert, 2008; Tak et al., 2009). Recreational noise exposure is more difficult to quantify; however, estimates suggest that exposures can often exceed 100 dB SPL (Clark, 1991). Previous evidence suggests that even moderate exposures over extended periods are neuropathic (Maison et al., 2013). Thus, in modern society, where noise exposure is common, it is plausible that interindividual variability in hearing ability may partly originate from hidden deficits caused by noise exposure or its interactions with aging and genetic susceptibility (Davis et al., 2003; Kujawa and Liberman, 2006). The correlations, albeit weak, between temporal coding measures and noise-exposure history support the notion that neuropathy may be present even in normalhearing listeners. Recent independent findings relating noiseexposure to EFR measures are consistent with this interpretation (Plack et al., 2014). Stone and Moore (2014) found that higher levels of recreational noise exposure in young listeners were associated with poorer AM detection thresholds, but only at lower sound levels. The low level effect is consistent with the loss of high-SR fibers; indeed, an $\sim 40 \%$ loss of overall synapse count (Lin et al., 2011a) accompanied by change in population statistics of high-SR/low-SR counts from $55 / 45$ to $\sim 75 / 25$ is consistent with some high-SR neuropathy being present (Fig. 2). That Stone and Moore (2014) did not see noise-exposure effects at higher sound levels may possibly be due to associated increases in absolute thresholds that they found; outer hair cell loss can cause broadened tuning and loudness recruitment leading to improved AM detection at higher levels (Moore et al., 1996). Additional studies are needed to clarify these issues and assess the sensitivity of behavioral and EFR-based approaches in revealing noise damage when multiple mechanisms are involved simultaneously.

In real-world situations, selective attention performance depends on many nonsensory factors such as language ability, 
memory, etc. (Conway et al., 2001; Surprenant and Watson, 2001; Kidd et al., 2007). However, in this study, we wanted to determine whether sensory coding fidelity of suprathreshold sound impacts the ability to communicate in everyday settings. Accordingly, we designed a spatial attention task where language and memory demands were low and sensory encoding limitations were the likely bottleneck on performance. Error patterns confirm that we achieved this goal. The fact that selective attention performance correlates with suprathreshold coding fidelity lends credence to the idea that neuropathy influences how well listeners can communicate in daily life. Although aging and noise exposure likely contribute to suprathreshold hearing deficits, inherent variability from genetic, epigenetic, and experiential factors no doubt also influence individual outcomes. Experienceand training-dependent long-term plasticity effects are known to affect processing at various stages of the auditory system and can improve behavioral performance in auditory tasks (Russo et al., 2005; Polley et al., 2006). Additional studies are needed to assess the contribution of such long-term plasticity to the differences in electrophysiological measures (such as the EFR) in the general $\mathrm{NH}$ population and to ascertain the degree to which such plasticity can ameliorate the effects of neuropathy. Individual differences in the olivocochlear efferent system and middle ear reflexes also must be examined (Backus and Guinan, 2007); however, the relationship between efferent effects and EFR measures is likely complicated given that (1) activation of the efferent system may help protect listeners from noise-induced cochlear neuropathy (Maison et al., 2013) and (2) low-SR nerve activity may be linked to the acoustic activation of the efferent system and the stapedius reflexes (Liberman, 1988; Kobler et al., 1992). Thus, once some degree of neurodegeneration occurs, it is possible that the efferent system may become less effective, leading to further damage. Additional studies are needed to understand the undoubtedly complex relationships between long-term plasticity, efferent function, middle-ear reflexes, and neuropathy.

We conclude that $\mathrm{NH}$ listeners from the general population vary significantly in how sensitive they are to suprathreshold temporal structure in sound. These differences appear to be unrelated to cochlear transduction, consistent with the insensitivity of the audiogram to such differences. Instead, results suggest that these differences come from the fidelity with which temporal features are encoded by very early levels of the neural pathway and are consistent with cochlear neuropathy degrading suprathreshold hearing ability in some listeners. These deficits may not be evident in quiet settings, but will affect how well listeners communicate in common social settings. In the field of hearing science, there is a growing belief that such hidden hearing loss is a underrecognized and poorly understood public-health concern. Hidden hearing loss has also been hypothesized to underlie tinnitus in listeners with normal audiograms (Schaette and McAlpine, 2011; Eggermont and Roberts, 2014; Plack et al., 2014). Thus there is tremendous interest in objective assessment tools for such deficits. Our results suggest that EFR-based measures are promising in this respect. Additional experiments are needed to develop targeted methods for the assessment, detailed characterization, and prevention of such hidden hearing loss.

\section{References}

Backus BC, Guinan JJ Jr (2007) Measurement of the distribution of medial olivocochlear acoustic reflex strengths across normal-hearing individuals via otoacoustic emissions. J Assoc Res Otolaryngol 8:484-496. CrossRef

Benjamini Y, Hochberg Y (1995) Controlling the false discovery rate: a practical and powerful approach to multiple testing. J Royal Stat Soc Series B Stat Methodol 57:289-300.
Bernstein LR, Trahiotis C (2002) Enhancing sensitivity to interaural delays at high frequencies by using "transposed stimuli." J Acoust Soc Am 112: 1026-1036. CrossRef

Bernstein LR, Trahiotis C (2008) Discrimination of interaural temporal disparities conveyed by high-frequency sinusoidally amplitude-modulated tones and high-frequency transposed tones: effects of spectrally flanking noises. J Acoust Soc Am 124:3088-3094. CrossRef

Bharadwaj HM, Shinn-Cunningham BG (2014) Rapid acquisition of auditory subcortical steady-state responses using multichannel recordings. Clin Neurophysiol 125:1878-1888. CrossRef

Bharadwaj HM, Verhulst S, Shaheen L, Liberman MC, Shinn-Cunningham BG (2014) Cochlear neuropathy and the coding of supra-threshold sound. Front Syst Neurosci 8:26. CrossRef Medline

Blauert J (1997) Spatial hearing: the psychophysics of human sound localization. Cambridge, MA: MIT.

Box GE, Tiao GC (2011) Bayesian inference in statistical analysis, Vol 40. New York: Wiley.

Chermak GD, Musiek FE (1997) Central auditory processing disorders: new perspectives. San Diego: Singular.

Christiansen C, MacDonald EN, Dau T (2013) Contribution of envelope periodicity to release from speech-on-speech masking. J Acoust Soc Am 134:2197-2204. CrossRef

Clark WW (1991) Noise exposure from leisure activities: a review. J Acoust Soc Am 90:175-181. CrossRef

Conway A, Cowan N, Bunting M (2001) The cocktail party phenomenon revisited: the importance of working memory capacity. Psychon Bull Rev 8:331-335. CrossRef

Cooper J Jr, Gates GA (1991) Hearing in the elderly-the Framingham cohort, 1983-1985: Part II. Prevalence of central auditory processing disorders. Ear Hear 12:304-311. CrossRef

Costalupes JA (1985) Representation of tones in noise in the responses of auditory nerve fibers in cats. I. Comparison with detection thresholds. J Neurosci 5:3261-3269. Medline

Darwin C (1997) Auditory grouping. Trends Cogn Sci 1:327-333. CrossRef Davis R, Kozel P, Erway L (2003) Genetic influences in individual susceptibility to noise: a review. Noise Health 5:19-28. Medline

Dubno J (1984) Effects of age and mild hearing loss on speech recognition in noise. J Acoust Soc Am 76:87-96. CrossRef

Eggermont JJ, Roberts LE (2014) Tinnitus: animal models and findings in humans. Cell Tissue Res. Advance online publication. doi:10.1007/s00441-0141992-8. CrossRef Medline

Fitzgibbons PJ, Gordon-Salant S (2010) Behavioral studies with aging humans: hearing sensitivity and psychoacoustics. In: The aging auditory system, pp 111-134. New York: Springer.

Furman AC, Kujawa SG, Liberman MC (2013) Noise-induced cochlear neuropathy is selective for fibers with low spontaneous rates. J Neurophysiol 110:577-586. CrossRef

Gatehouse S, Noble W (2004) The speech, spatial and qualities of hearing scale (SSQ). Int J Audiol 43:85-99. CrossRef

Grose J, Mamo S (2010) Processing of temporal fine structure as a function of age. Ear Hear 31:755-760. CrossRef

Hadfield JD (2010) MCMC methods for multi-response generalized linear mixed models: the MCMCglmm R package. J Stat Softw 33:1-22.

Herdman AT, Lins O, Van Roon P, Stapells DR, Scherg M, Picton TW (2002) Intracerebral sources of human auditory steady-state responses. Brain Topogr 15:69-86. CrossRef

Hind SE, Haines-Bazrafshan R, Benton CL, Brassington W, Towle B, Moore DR (2011) Prevalence of clinical referrals having hearing thresholds within normal limits. Int J Audiol 50:708-716. CrossRef

Jääskeläinen IP, Ahveninen J, Bonmassar G, Dale AM, Ilmoniemi RJ, Levänen S, Lin F-H, May P, Melcher J, Stufflebeam S, et al. (2004) Human posterior auditory cortex gates novel sounds to consciousness. Proc Natl Acad Sci U S A 101(17):6809-6814. CrossRef

Johnson DH (1980) The relationship between spike rate and synchrony in responses of auditory-nerve fibers to single tones. J Acoust Soc Am 68: 1115-1122. CrossRef

Johnson TA, Neely ST, Garner CA, Gorga MP (2006) Influence of primarylevel and primary-frequency ratios on human distortion product otoacoustic emissions. J Acoust Soc Am 119:418-428. CrossRef

Joris PX, Yin TC (1992) Responses to amplitude-modulated tones in the auditory nerve of the cat. J Acoust Soc Am 91:215-232. CrossRef

Joris PX, Carney LH, Smith PH, Yin TC (1994) Enhancement of neural 
synchronization in the anteroventral cochlear nucleus I. Responses to tones at the characteristic frequency. J Neurophysiol 71:1022-1036. Medline

Kidd G, Watson C, Gygi B (2007) Individual differences in auditory abilities. J Acoust Soc Am 122:418-435. CrossRef

Kiren T, Aoyagi M, Furuse H, Koike Y (1994) An experimental study on the generator of amplitude-modulation following response. Acta Otolaryngol Suppl 511:28-33. Medline

Kobler J, Guinan JJ Jr, Vacher S, Norris B (1992) Acoustic reflex frequency selectivity in single stapedius motoneurons of the cat. J Neurophysiol 68:807-817. Medline

Kujawa S, Liberman M (2009) Adding insult to injury: Cochlear nerve degeneration after "temporary" noise-induced hearing loss. J Neurosci 29: 14077-14085. CrossRef

Kujawa SG, Liberman MC (2006) Acceleration of age-related hearing loss by early noise exposure: evidence of a misspent youth. J Neurosci 26: 2115-2123. CrossRef

Kumar G, Amen F, Roy D (2007) Normal hearing tests: is a further appointment really necessary? J R Soc Med 100:66. CrossRef

Kuss M, Jäkel F, Wichmann FA (2005) Bayesian inference for psychometric functions. J Vis 5(8):478-492. CrossRef Medline

Kuwada S, Anderson JS, Batra R, Fitzpatrick DC, Teissier N, D’Angelo WR (2002) Sources of the scalp-recorded amplitude modulation following response. J Am Acad Audiol 13:188-204. Medline

Liberman MC (1988) Physiology of cochlear efferent and afferent neurons: direct comparisons in the same animal. Hear Res 34:179-191. CrossRef

Lin FR, Niparko JK, Ferrucci L (2011a) Hearing loss prevalence in the United States. Arch Intern Med 171:1851-1853. CrossRef

Lin HW, Furman AC, Kujawa SG, Liberman MC (2011b) Primary neural degeneration in the Guinea pig cochlea after reversible noise-induced threshold shift. J Assoc Res Otolaryngol 12:605-616. CrossRef

Lobarinas E, Salvi R, Ding D (2013) Insensitivity of the audiogram to carboplatin induced inner hair cell loss in chinchillas. Hear Res 302:113-120. CrossRef Medline

Lopez-Poveda EA, Barrios P (2013) Perception of stochastically undersampled sound waveforms: a model of auditory deafferentation. Front Neurosci 7:124. CrossRef

Maison SF, Usubuchi H, Liberman MC (2013) Efferent feedback minimizes cochlear neuropathy from moderate noise exposure. J Neurosci 33:55425552. CrossRef

Makary CA, Shin J, Kujawa SG, Liberman MC, Merchant SN (2011) Agerelated primary cochlear neuronal degeneration in human temporal bones. J Assoc Res Otolaryngol 12:711-717. CrossRef

Moore BC (2007) Cochlear hearing loss: physiological, psychological and technical issues, Ed 2. New York: Wiley.

Moore BC (2008) The role of temporal fine structure processing in pitch perception, masking, and speech perception for normal-hearing and hearing-impaired people. J Assoc Res Otolaryngol 9:399-406. CrossRef

Moore BC, Wojtczak M, Vickers DA (1996) Effect of loudness recruitment on the perception of amplitude modulation. J Acoust Soc Am 100:481489. CrossRef

Oxenham AJ, Shera CA (2003) Estimates of human cochlear tuning at low levels using forward and simultaneous masking. J Assoc Res Otolaryngol 4:541-554. CrossRef

Patterson RD (1976) Auditory filter shapes derived with noise stimuli. J Acoust Soc Am 59:640 -654. CrossRef

Picton TW, John MS, Dimitrijevic A, Purcell D (2003) Human auditory steady-state responses. Int J Audiol 42:177-219. CrossRef

Pierson LL, Gerhardt KJ, Rodriguez GP, Yanke RB (1994) Relationship between outer ear resonance and permanent noise-induced hearing loss. Am J Otolaryngol 15:37-40. CrossRef

Pinheiro J, Bates D (2000) Mixed-effects models in S and S-PLUS. New York: Springer.

Plack CJ, Barker D, Prendergast G (2014) Perceptual consequences of "hidden" hearing loss. Trends Hear 18:2331216514550621. CrossRef Medline

Polley DB, Steinberg EE, Merzenich MM (2006) Perceptual learning directs auditory cortical map reorganization through top-down influences. J Neurosci 26:4970-4982. CrossRef

Ruggles DR, Bharadwaj HM, Shinn-Cunningham BG (2012) Why middle- aged listeners have trouble hearing in everyday settings. Curr Biol 22: 1417-1422. CrossRef

Ruggles D, Bharadwaj H, Shinn-Cunningham B (2011) Normal hearing is not enough to guarantee robust encoding of suprathreshold features important in everyday communication. Proc Natl Acad Sci U S A 108: 15516-15521. CrossRef Medline

Russo N, Nicol T, Zecker S, Hayes E, Kraus N (2005) Auditory training improves neural timing in the human brainstem. Behav Brain Res 156: 95-103. CrossRef

Salminen NH, Tiitinen H, Yrttiaho S, May PJ (2010) The neural code for interaural time difference in human auditory cortex. J Acoust Soc Am 127:EL60-EL65. CrossRef Medline

Saunders G, Haggard M (1992) The clinical assessment of "obscure auditory dysfunction" (OAD) 2. Case control analysis of determining factors. Ear Hear 13:241-254. CrossRef

Schaette R, McAlpine D (2011) Tinnitus with a normal audiogram: physiological evidence for hidden hearing loss and computational model. J Neurosci 31(38):13452-13457. CrossRef

Schmiedt R, Mills J, Boettcher F (1996) Age-related loss of activity of auditory-nerve fibers. J Neurophysiol 76:2799-2803. Medline

Schuknecht HF, Woellner RC (1955) An experimental and clinical study of deafness from lesions of the cochlear nerve. J Laryngol Otol 69:75-97. CrossRef

Sergeyenko Y, Lall K, Liberman MC, Kujawa SG (2013) Age-related cochlear synaptopathy: an early-onset contributor to auditory functional decline. J Neurosci 33:13686-13694. CrossRef

Shamma SA, Elhilali M, Micheyl C (2011) Temporal coherence and attention in auditory scene analysis. Trends Neurosci 34:114-123. CrossRef

Snell KB, Frisina DR (2000) Relationships among age-related differences in gap detection and word recognition. J Acoust Soc Am 107:1615-1626. CrossRef

Snell K, Mapes F, Hickman E, Frisina D (2002) Word recognition in competing babble and the effects of age, temporal processing, and absolute sensitivity. J Acoust Soc Am 112:720-727. CrossRef

Steiger JH (1980) Tests for comparing elements of a correlation matrix. Psychol Bull 87:245. CrossRef

Stone MA, Moore BC (2014) Amplitude-modulation detection by recreational-noise-exposed humans with near-normal hearing thresholds and its medium-term progression. Hear Res 317:50-62. CrossRef Medline

Strelcyk O, Dau T (2009) Relations between frequency selectivity, temporal fine-structure processing, and speech reception in impaired hearing. J Acoust Soc Am 125:3328-3345. CrossRef

Surprenant A, Watson C (2001) Individual differences in the processing of speech and nonspeech sounds by normal-hearing listeners. J Acoust Soc Am 110:2085-2095. CrossRef

Tak S, Calvert GM (2008) Hearing difficulty attributable to employment by industry and occupation: an analysis of the National Health Interview Survey-United States, 1997 to 2003. J Occup Environ Med 50:46-56. CrossRef

Tak S, Davis RR, Calvert GM (2009) Exposure to hazardous workplace noise and use of hearing protection devices among US workersNHANES, 1999-2004. Am J Indust Med 52:358-371. CrossRef

Viemeister NF (1979) Temporal modulation transfer functions based upon modulation thresholds. J Acoust Soc Am 66:1364-1380. CrossRef

Yost W (2007) The abnormal auditory system. In: Fundamentals of hearing: an introduction, Ed 5. San Diego: Academic.

Zeng F, Nie K, Stickney G, Kong Y, Vongphoe M, Bhargave A, Wei C, Cao K (2005) Speech recognition with amplitude and frequency modulations. Proc Natl Acad Sci U S A 102:2293-2298. CrossRef

Zhao F, Stephens D (1996) Hearing complaints of patients with KingKopetzky syndrome (obscure auditory dysfunction). Br J Audiol 30:397402. CrossRef

Zhu L, Bharadwaj H, Xia J, Shinn-Cunningham B (2013) A comparison of spectral magnitude and phase-locking value analyses of the frequencyfollowing response to complex tones. J Acoust Soc Am 134:384-395. CrossRef

Zilany MSA, Bruce IC, Carney LH (2014) Updated parameters and expanded simulation options for a model of the auditory periphery. J Acoust Soc Am 135:283-286. CrossRef 